\title{
A Distributed Demand Side Energy Management Algorithm for Smart Grid
}

\author{
Min-fan He ${ }^{1}$, Fu-xing Zhang ${ }^{2} \oplus$, Yong Huang ${ }^{1}$, Jian Chen ${ }^{1}$, Jue Wang ${ }^{3}$ and Rui Wang ${ }^{4, *}$ \\ 1 School of Mathematics and Big Data, Foshan University, Foshan 528000, China; \\ heminfan1980@126.com (M.H.); hyhy1223@hotmail.com (Y.H.); chenjian704@163.com (J.C.) \\ 2 State Grid Key Laboratory of Information \& Network Security, Global Energy Interconnection Research \\ Institute, Beijing 102211, China; zfx@nudt.edu.cn \\ 3 School of Physics \& information technology, Shaanxi Normal University, Xi'an, 710119 China; \\ yuyuwangnormalsx@sina.cn \\ 4 National University of Defense Technology, Changsha, 410073, China \\ * Correspondence: ruiwangnudt@gmail.com; Tel.: +86-188-7496-2006
}

Received: 9 January 2019; Accepted: 26 January 2019; Published: 29 January 2019

Abstract: This paper proposes a model predictive control (MPC) framework-based distributed demand side energy management method (denoted as DMPC) for users and utilities in a smart grid. The users are equipped with renewable energy resources (RESs), energy storage system (ESSs) and different types of smart loads. With the proposed method, each user finds an optimal operation routine in response to the varying electricity prices according to his/her own preference individually, for example, the power reduction of flexible loads, the start time of shift-able loads, the operation power of schedulable loads, and the charge/discharge routine of the ESSs. Moreover, in the method a penalty term is used to avoid large fluctuation of the user's operation routines in two consecutive iteration steps. In addition, unlike traditional energy management methods which neglect the forecast errors, the proposed DMPC method can adapt the operation routine to newly updated data. The DMPC is compared with a frequently used method, namely, a day-ahead programming-based method (denoted as DDA). Simulation results demonstrate the efficiency and flexibility of the DMPC over the DDA method.

Keywords: model predictive control (MPC); smart grid; demand side management (DSM); distributed optimization; game theory

\section{Introduction}

In recent years, the smart grid has undergone significant innovation and moved from conceptual to operational. It has been repeatedly demonstrated to provide more reliable, environment-friendly and economically efficient power systems [1].

With the development of advanced information and communication technologies and smart metering infrastructures, the two-way digital communication system now enables the utility company to share information, e.g., the time-dependent electricity price, with users. Moreover, the users can adopt related adjustment strategies to minimize their operation cost, and further to improve the overall energy system performance [2]. This is referred to as demand-side management (DSM) [3,4], which has attracted more and more attention.

Usually, candidate control actions that a user can perform include, for example, delaying the start time of shift-able loads, decreasing the demand of power flexible loads, modifying the power schedule of schedulable loads, ESS charging/discharging, selling extra electricity (produced by distributed wind and/or PV generators) to utility companies. By the DSM, a "win-win" situation between users and utility companies is expected [5]. 
To date, several effective DSM algorithms have been proposed [6,7], which range from improving energy efficiency by using better materials to coordinating operation strategies of different appliances, distributed energy resources (DERs) such as renewable energy resources (RESs) and energy storage systems (ESSs).

However, existing methods, as will be reviewed in Section 2, are either not efficient or have not considered all related factors, e.g., the application of DERs, the forecast uncertainties. This study therefore proposes a model-predicative control (MPC)-based distributed DSM method (DMPC) for smart grid, aiming to handle the mentioned issue, as well as providing a more realistic energy management model.

The proposed algorithm integrates newly updated forecast of RESs and loads to minimize the disturbance. Its feedback mechanism is well-suited to deal with forecast uncertainties [8,9]. Specifically, in the DMPC each user is equipped with an energy management system (EMS) to control his/her own dispatchable units (such as the ESS, power flexible loads, shift-able loads and schedulable loads), to communicate with utility companies, and to coordinate with other users so as to minimize his/her own cost and maximize the social welfare. Moreover, in the DMPC, the user needs not to broadcast his/her own energy schedule to other users like many existing studies, e.g., [10,11]. The user only needs to send his/her own load demand routine to the utility company. This can not only protect user privacy, but also reduce the communication burden. In addition, in the DMPC, a penalty term is adopted in the cost function to confine the power changes in two consecutives iterations. Furthermore, the discomfort penalty caused by curtailing the power flexible loads, delaying the shift-able loads and changing the operation schedule of schedulable loads is also considered. The user preferences are also considered. All these factors enable the proposed DMPC method to be more realistic and applicable. Overall, the main contributions of this study are as follows:

1. A novel DSM is proposed that considers minimizing the operation cost of each user as well as the discomfort caused by the change of load operation schedule. The DSM can also accommodate different types of user-preferences.

2. A distributed optimization algorithm based on games theory is proposed to coordinate the users' operation schedules to minimize their own operation costs. Meanwhile, an iteration mechanism is proposed to accelerate the convergence speed.

3. An MPC framework is implemented to integrate the user operation management model and distributed optimization algorithm. The MPC framework, featuring a rolling up and feedback mechanism, is shown to be able to handle the negative impacts caused by the forecast uncertainty of the RESs output and load demand.

The rest of this paper is organized as follows. Section 2 reviews a selection of representative works that are related. Section 3 presents the model formulation of the smart grid, including the system overall model, the model of components such as the smart loads, the ESS, the pricing policy, and the operation optimization objectives for each user. Section 4 introduces the MPC-based distributed control scheme to coordinate the operations of all users. Numerical simulations are presented in Section 5. Section 6 concludes the paper.

\section{Literature Review}

There have been several studies investigating the DSM for smart grid. These methods have their own merits and disadvantages. Next, we review a selection of representative ones.

Since large commercial or industrial centers usually have large energy consumption, Aalami, et al. [12] discussed the DSM for large users. Setlhaolo, et al. [13] further studied the optimal scheduling scheme for typical home appliances. Zhang et al. [14] investigated the energy management strategy of a smart home with the consideration of the demand response (DR). Erdinc [15] evaluated the economic impacts of the ESS, distributed generators (DG) and shift-able loads under different DR strategies. These works consider DSM for either large aggregators or a single user only. Also, 
centralized energy management strategies are implemented to obtain the control sequence with the consideration of constant electricity price. These early methods obviously lose effect in practice, as the electricity price changes as the operation schedule of the users changes.

To tackle the above issue, Mohsenian, et al. [16] presented an autonomous and distributed DSM system in which a sequential energy scheduling strategy is obtained using a games theory model. However, the method requires a priori knowledge of the start and end time of all appliances for each user. All users have to broadcast their total load schedule to the others. Hazem and Alberto [10] studied the DSM problem for users equipped with ESS. Two games are included in the DSM problem: the first one is based on a non-cooperative games theory, and is implemented among users. The other is a stackelberg games theory-based model that is implemented between users and the utility company. Results show that by the DSM strategy the peak-to-average power ratio is reduced significantly. With a real-time DSM, Shengrong and Richard [11] proposed a four-stage game-theoretical decision-making scheme for electricity retailer and energy users. In the first three stages, the electricity retailer acts as the game leader to determine how much electricity should be produced and how much the electricity price should be set to maximize its profit. In the fourth stage, the users adjust their electricity load demand individually to maximize their own utility company.

Though the above methods can guarantee the equilibrium of distributed optimization, their efficiency is low due to the use of sequential-based games theory, requiring many iterations to reach the optima. Moreover, these methods broadcast the total load demand schedule of all users, which are prone to release privacy of users, and causes extra communication burden.

Song et al. [17] proposed a non-stationary DSM mechanism that a repeated game framework was integrated to minimize the overall cost of power consummation and discomfort. A critical peak price scheme is utilized and the incentive-compatible is discussed in respect of a long-term run operation cost. Fathi and Bevrani [18] discussed the appliances energy consumption scheduling for a home energy management system with non-cooperative game theory to find the optimal start time and operation mode of user appliances in response to the varying electricity price. Tan et al. [19] analyzed the DSM for smart grids integrated plug-in electric vehicles. The fluctuant electricity price and games among users were considered.

Although the above works have provided some effective strategies, the DERs, which are important units for the future smart grids, are not considered. Some studies, e.g., [20-22], have considered DERs. Specifically, Atzeni et al. [20] proposed a DSM method consisted of a day-ahead optimization process that corresponded to energy production and energy storage scheduling. The non-cooperative game and cooperative game scenarios were both discussed to optimize the production/storage strategies for users. Peng et al. [21] proposed a parallel autonomous optimization scheme for DSM in smart grids that integrated RESs generators. Kamyab et al. [22] studied the interaction among multiple utility companies and multiple users in smart grid using two non-cooperative games models. However, the forecast uncertainty of RESs output is not considered. It is known that forecast errors might lead to significantly hamper outcomes [23].

Thus, Wang et al. [24] proposed a control strategy to coordinate the operation of networked microgrids in a smart distribution system. Specifically, a stochastic bi-level game theory model is implemented to achieve the equilibrium among all users. The uncertainties of the DG outputs are considered with a stochastic programming model. However, as the penetration level of DERs becomes high, the stochastic-based open-loop energy management strategy lose effect due to the negative influence introduced by the forecast uncertainties of RESs. Stephens et al. [25] then proposed a game theoretic model predictive control (MPC) approach for DSM which could adapt to real-time data. Simulation results show that this method is more effective than [24] when the penetration level of RESs is high. However, the energy user model in [25] is simple and the discomfort penalty cost is not considered.

Overall, although several DSM methods have been shown to be effective under certain situations, none of existing models considers all factors, e.g., the forecast uncertainties of RESs output, the 
discomfort cost as well as different user preferences, altogether, which has greatly limited the application of existing methods.

\section{System Model and Problem Formulation}

This study considers a smart grid consisting of a utility company and multiple $(M)$ energy users as shown in Figure 1. For brevity, utility company is abbreviated as utility in the following sections. A DSM control center is deployed in the utility and an EMS is deployed on each user's side. The EMS has the following functions: (i) communicating with the smart meters in the user side and the DSM control center in utility; (ii) predicating the power generations of RESs and load demand; (iii) optimizing operation routines of the dispatchable units of the user based on forecasts, electricity price and so on. The control orders for dispatchable units will be implemented through the programmable logic controller (PLC) in the system. For each user, he/she cannot only purchase electricity energy from the utility but also sell extra power generation back to the utility to make revenue. Moreover, for users with an ESS unit, both the selling time and the amount of selling power can be optimized.

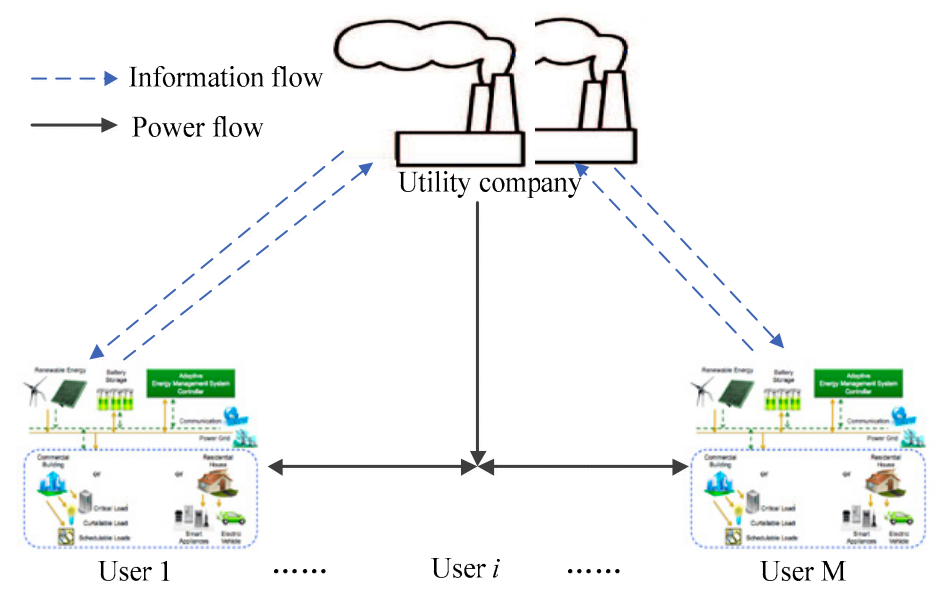

Figure 1. Power and information flows among users and the utility.

Prior to describing the system model, it is assumed that the whole control and prediction horizon is divided into $T$ time intervals. Duration of each time interval is $\Delta t$, denoted as $t \in[1,2, \cdots, T]$. $M$ energy users are considered, denoted as $i \in[1,2, \cdots, M]$.

\subsection{Model of Loads}

According to the feature of different loads, they are classified as base loads, power flexible loads, shift-able loads and schedulable loads $[26,27]$. Therefore, the total load demand in period $t$ is computed as Equation (1). Also, the forecast error of load demand is assumed to fit the Gaussian distribution [28].

$$
l_{i}(t)=l_{i, B}(t)+\sum_{f=1}^{F} l_{i, f}(t) \cdot\left(1-\theta_{i, f}(t)\right)+\sum_{a=1}^{A_{i, S f}} l_{i, a} \cdot \delta_{i, a}(t)+\sum_{b=1}^{A_{i, S e}} l_{i, b}(t)
$$

For based load, the operation time and power demand must be satisfied, and cannot be changed. The power demand cannot excess the defined capacity limit.

$$
0 \leq l_{i, B}(t) \leq l_{i, B}^{\max }
$$

For flexible loads, the power demand can be curtailed by users to save money or to use in emergency conditions. However, the curtailed power cannot exceed a certain range to avoid the 
dissatisfaction caused by adjustment actions. In the model, $F_{i}$ power flexible appliances are considered, denoted as $f \in\left[1,2, \cdots, F_{i}\right]$.

$$
0 \leq \theta_{i, f}(t) \leq \theta_{i, f}^{\max }
$$

For shift-able load, the start time can be delayed in a certain time window, but its operation power is fixed and cannot be adjusted. Also, once the task is started, it cannot be stopped before the task is completed. In the model, $A_{i}$ power flexible appliances are considered, denoted as $a \in\left[1,2, \cdots, A_{i}\right]$.

$$
\begin{gathered}
\delta_{i, a}(t)=\left\{\begin{array}{c}
0 \quad \text { if } t<T_{i, a}^{\text {start }} \\
0 \quad \text { if } t>T_{i, a}^{\text {end }} \\
0 / 1 \quad \text { otherwise }
\end{array}, a \in\left[1,2, \cdots, A_{i}\right]\right. \\
\sum_{T_{i, a}^{\text {start }}}^{T_{i, a}^{\text {end }}} \delta_{i, a}(t)=T_{i, a} \\
\sum_{t}^{T_{i, a}+t} \delta_{i, a}(t) \geq T_{i, a}\left(\delta_{i, a}(t)-\delta_{i, a}(t-1)\right), t \in\left[T_{i, a}^{\text {start }}, T_{i, a}^{\text {end }}\right]
\end{gathered}
$$

For schedulable loads, both the start time and operation power can be adjusted if the total demand is satisfied before the deadline. However, once the load is started it should be operated till the task is completed. In this model, $B_{i}$ power flexible appliance is considered, denoted as $b \in\left[1,2, \cdots, B_{i}\right]$.

$$
\begin{gathered}
l_{i, b}^{\min } \delta_{i, b}(t) \leq l_{i, b}(t) \leq l_{i, b}^{\max } \delta_{i, b}(t) \\
\sum_{t}^{T_{i, a}+t} l_{i, b}(t) \Delta t=E_{i, b} \\
\sum_{t}^{T_{i, b}+t} \delta_{i, b}(t) \geq T_{i, b}\left(\delta_{i, b}(t)-\delta_{i, b}(t-1)\right), t \in\left[T_{i, b}^{\text {start }}, T_{i, b}^{\text {end }}\right]
\end{gathered}
$$

Next, we model the discomfort cost. Specifically, the discomfort $\operatorname{cost} C_{i, f}(t)$ by power reduction of power flexible loads $f$ is modelled as a convex non-decreasing function, as shown in Equation (10).

$$
C_{i, f}(t)=l_{i, f}(t) \theta_{i, f}(t) c_{i, f}^{\text {curt }} \Delta t
$$

The discomfort $\operatorname{cost} C_{i, a}(t)$ by the delay of the start time of shift-able load $a$ is computed as in Equation (11). It indicates that as the delay start time increases, more discomfort penalty cost must be paid.

$$
C_{i, a}(t)=c_{i, a}^{s f}\left(\delta_{i, a}(t)-\delta_{i, a}^{b a s e}(t)\right)^{2}
$$

where $\delta_{i, a}^{b a s e}(t)$ is the user's baseline on/off status of appliance $a$ in period $t$.

The discomfort cost $C_{i, b}(t)$ caused by appliances, e.g., $b$ in period $t$ comes from two parts: the cost of power adjustment $\operatorname{cost} C_{i, b}^{p o}(t)$, and the cost induced by the delay of start time $C_{i, b}^{t m}(t)$.

$$
\begin{gathered}
C_{i, b}(t)=C_{i, b}^{p o}(t)+C_{i, b}^{t m}(t) \\
C_{i, b}^{p o}=c_{i, b}^{p}\left|l_{i, b}(t)-l_{i, b}^{b a s e}(t)\right| \Delta t \\
C_{i, b}^{t m}=c_{i, b}^{t}\left(\delta_{i, b}(t)-\delta_{i, b}^{\text {base }}(t)\right)^{2}
\end{gathered}
$$

where $\delta_{i, b}^{\text {base }}(t), l_{i, b}^{\text {base }}(t)$ denote the user's baseline on/off status and power demand of appliance $a$ in period $t$, respectively. 


\subsection{Model of DERs}

The application of distributed energy resources (DERs) including RESs and ESSs has attracted more and more attention due to the increase of energy demand and emissions of greenhouse gases.

Despite the advantages of RESs, the randomness and fluctuation of the RESs power generation make their applications in smart grid difficult. To guarantee safe use of RESs, their power outputs are constrained within a certain range.

$$
\begin{gathered}
0 \leq P_{i, P V}(t) \leq P_{i, P V}^{\max } \\
0 \leq P_{i, \text { wind }}(t) \leq P_{i, \text { wind }}^{\text {max }}
\end{gathered}
$$

Similarly, the forecast errors of wind and PV generation is described by Gaussian distributions [29, 30].

As the most flexible unit in smart grid, the ESS model mainly consists of the system dynamic model, energy capacity model, power charge/discharge model and operation status model.

$$
\begin{gathered}
E_{i, E S S}(t+1)=E_{i, E S S}(t)+\eta_{i, E S S c} P_{i, E S S c}(t) \Delta t-1 / \eta_{i, E S S d} P_{i, E S S d}(t) \Delta t-\varepsilon_{i, E S S} \\
E_{i, E S S}^{\min } \leq E_{i, E S S}(t+1) \leq E_{i, E S S}^{\max } \\
\delta_{i, E S S c}(t) P_{i, E S S c}^{\min } \leq P_{i, E S S c}(t) \leq \delta_{i, E S S c}(t) P_{i, E S S c}^{\max } \\
\delta_{i, E S S d}(t) P_{i, E S S d}^{\min } \leq P_{i, E S S d}(t) \leq \delta_{i, E S S d}(t) P_{i, E S S d}^{\max } \\
\delta_{i, E S S c}(t)+\delta_{i, E S S d}(t) \leq 1
\end{gathered}
$$

The initial energy level is denoted as $E_{i, \mathrm{ESS}}^{i n i t}$, it satisfies $E_{i, \mathrm{ESS}}^{\text {init }}=E_{i, E S S}(1)$. To effectively handle emergency conditions, the energy level of the ESS is reset to near the half energy capacity at the beginning of each day. Moreover, the operation and maintenance cost of ESS [31] is calculated as follows.

$$
C_{i, E S S}(t)=c_{i, E}^{O \& M}\left(P_{i, E c}(t)+P_{i, E d}(t)\right) \Delta t
$$

\subsection{Energy Price Model}

The base price $p_{u}(t)$ comes from the cost by thermal generators (we consider RESs all are deployed in the user side, and they are not the main power source) or a simple artificial cost tariff which is used by the utility to impose a proper DSM program.

As the price function is non-decreasing convex, we set the time-dependent base price function to be proportional to the first order derivative of the time-dependent generation cost [32].

$$
p_{u}(t)=\nabla C_{u}\left(P_{u}(t)\right)
$$

where $P_{u}(t)$ is the power generated by the utility, which equals to the summary of the load demands for all users.

$$
P_{u}(t)=\sum_{i=1}^{M}\left(l_{i}(t)-P_{i, P V}(t)-P_{i, \text { wind }}(t)+P_{i, E S S c}(t)-P_{i, E S S d}(t)\right)
$$

To promote the use of DERs as well as reduce the negative impacts of randomness of the RESs outputs, the selling electricity price $p_{i, s}(t)$ for users is set lower than the base price [31]. Meanwhile, according to the rate-of-return regulations [33], the buying electricity price $p_{i, b}(t)$ for users is set higher than the base price.

$$
\begin{aligned}
& p_{i, b}(t)=\rho_{i, b}(t) p_{u}(t) \\
& p_{i, S}(t)=\rho_{i, s}(t) p_{u}(t)
\end{aligned}
$$

where $\rho_{i, b}(t)>1>\rho_{i, s}(t)$. 
The real-time electricity price mechanism defined in Equations (25) and (26) guarantees the user's benefits, that is, when the base price is high the selling price is also high. Meanwhile, the difference between the buying price and the selling price promote the users to consume the power generated by themselves, and to encourage the utility to buy energy from the users.

Furthermore, to reduce the negative impacts introduced by the intermittent and random RESs outputs to the power system, users are not allowed to sell energy back to the utility if the forecasted RESs generation cannot supply the energy demand of the user completely.

Without loss of the generality, we set the operation $\operatorname{cost} C_{u}\left(P_{u}(t)\right)$ as,

$$
C_{u}\left(P_{u}(t)\right)=c_{1} P_{u}(t)+c_{2}\left(P_{u}(t)\right)^{2}
$$

where $c_{1}, c_{2}$ are constants.

\subsection{Power Interaction Model}

Because of Equations (25) and (26), the buying/selling energy cost model for each user is not a continuous model, becoming a mixed logical dynamic (MLD) model [14].

The power interaction between users and utility is modelled as follows.

$$
\begin{gathered}
0 \leq P_{i, g I}(t) \leq P_{i, g I}^{\max } \delta_{i, g I}(t) \\
0 \leq P_{i, g O}(t) \leq P_{i, g O}^{\max } \delta_{i, g O}(t) \\
\delta_{i, g I}(t)+\delta_{i, g O}(t) \leq 1
\end{gathered}
$$

Equations (28)-(39) indicate that the buying and selling power of each user cannot exceed its capacity. Equation (30) indicates that a user cannot purchase and sell power at the same time.

The power flow in each user is shown as follows.

$$
l_{i}(t)+P_{i, E S S c}(t)+P_{i, g O}(t)=P_{i, E S S d}(t)+P_{i, g I}(t)+P_{i, P V}(t)+P_{i, w i n d}(t)
$$

Overall, Equation (24) is modified as follows.

$$
P_{u}(t)=\sum_{i=1}^{M} P_{i, I}(t)-\sum_{i=1}^{M} P_{i, O}(t)
$$

\subsection{Cost Model}

The overall cost $\Psi_{i}(t)$ as shown in Equation (33) consists of energy billing cost and the discomfort cost. The billing cost includes the energy purchasing cost, the energy selling revenue and the ESS operation cost. The discomfort cost includes the penalty cost of curtailing flexible loads, the delaying penalty cost of shift-able loads start time, and the adjustment penalty cost of schedulable loads.

$$
\Psi_{i}=\sum_{t=1}^{T} P_{i, g I}(t) p_{i, b}(t) \Delta t-\sum_{t=1}^{T} P_{i, g O}(t) p_{i, S}(t) \Delta t+\sum_{t=1}^{T} C_{i, E S S}(t)+\sum_{t=1}^{T} \sum_{f=1}^{F_{i}} C_{i, f}(t)+\sum_{t=1}^{T} \sum_{a=1}^{A_{i}} C_{i, a}(t)+\sum_{t=1}^{T} \sum_{b=1}^{B_{i}} C_{i, b}(t)
$$

$$
\text { Subject to (1)-(32) }
$$

The cost $\Psi_{u}$ for the utility includes the fuel consumption cost and the buyback cost from the users, which is computed as follows.

$$
\Psi_{u}=\sum_{t=1}^{T} C_{u}(t)+\sum_{t=1}^{T} \sum_{i=1}^{M} P_{i, O}(t) p_{i, s}(t) \Delta t
$$


According to Equations (33) and (34), the cost optimization model for users is a mixed integer programming (MIP) problem, it is non-convex. The scheme of each user is affected by other users since the electricity price is determined by the total load demand of the smart grid. To solve the centralized optimization problem, all users' specifications and preferences are required, which is often difficult to obtain in practice, considering the privacy protection and computation burden. Meanwhile, each user hopes to minimize his/her own operation cost. This is difficult to achieve in a centralized way. Alternatively, a reasonable way is to allow each user to optimize his/her own operation schedule in a distributed way

\section{MPC Based Distributed User Energy Management Strategy}

This section first presents the distributed optimization algorithm used to solve the cost optimization model and verifies the equilibrium of the proposed distributed algorithm. Second, the detailed process of the MPC-based distributed optimization method is described.

\subsection{Distributed User Energy Scheduling Optimization}

Game theory is adopted to capture the competition among users. All users are considered to be rational entities that are only interested in minimizing their own cost. Specifically, the game theory-based method is described as follows.

(i) Players: all users ( $M$ users) in the smart grid.

(ii) Strategies: each user $i \in M$ selects its strategy by scheduling the dispatchable units (smart loads and ESS) to minimize his/her own cost.

(iii) Payoffs: the payoff $P\left(y_{i}, y_{-i}\right)$ for user $i$ comprises two parts, see Equation (35): the actual operation cost $\Psi_{i}^{k}$ described in Equation (33) at iteration $k$, and the penalty cost $\Phi_{i}^{k}$ caused by large fluctuant of the operation routine in two successive iterations.

$$
\begin{gathered}
P\left(y_{i}, y_{-i}\right)=\Psi_{i}^{k}+\Phi_{i}^{k} \\
\Phi_{i}^{k}=\lambda_{i}^{k}\left(\sum_{t=1}^{T} \quad\left|\left(P_{i, E S S d}^{k}(t)-P_{i, E S S c}^{k}(t)\right)-\left(P_{i, E S S d}^{k-1}(t)-P_{i, E S S c}^{k-1}(t)\right)\right|\right. \\
+\sum_{t=1}^{T}\left|\left(P_{i, g I}^{k}(t)-P_{i, g O}^{k}(t)\right)-\left(P_{i, g I}^{k-1}(t)-P_{i, g O}^{k-1}(t)\right)\right| \\
\left.+\sum_{t=1}^{T} \sum_{a=1}^{A_{i}}\left|l_{i, a}^{k}(t)-l_{i, a}^{k-1}(t)\right|+\sum_{t=1}^{T} \sum_{b=1}^{B_{i}}\left|l_{i, b}^{k}(t)-l_{i, b}^{k-1}(t)\right|\right)
\end{gathered}
$$

Please note that we use $X_{i}^{k}$ to denote $X_{i}$ in the $k^{\text {th }}$ iteration, where $X$ can represent all the decision variables and objective functions in this paper.

Different from [21], the power varying of dispatchable units in two successive iterations is considered in this study. This is to avoid the situation that little difference for the power interaction but a large difference for the payoff in two successive iterations. Furthermore, Equation (36) can effectively cope with the power varying introduced by ESS. The detail distributed optimization process is shown in Table 1.

$$
\begin{gathered}
\nabla C_{o p t}=\sum_{i=1}^{M}\left|\Psi_{u}^{k}-\Psi_{u}^{k-1}\right| \\
\nabla L_{s h f}=\sum_{i=1}^{M} \sum_{t=1}^{T} \sum_{a=1}^{A_{i}}\left|l_{i, a} \delta_{i, a}^{k}(t)-l_{i, a} \delta_{i, a}^{k}(t)\right| \\
\nabla L_{s c h}=\sum_{i=1}^{M} \sum_{t=1}^{T} \sum_{b=1}^{B_{i}}\left|l_{i, b}^{k}(t)-l_{i, b}^{k-1}(t)\right| \\
\nabla P_{E S S}=\sum_{i=1}^{M} \sum_{t=1}^{T}\left|\left(P_{i, E S S d}^{k}(t)-P_{i, E S S c}^{k}(t)\right)-\left(P_{i, E S S d}^{k-1}(t)-P_{i, E S S c}^{k-1}(t)\right)\right|
\end{gathered}
$$




$$
\nabla P_{\text {grid }}=\sum_{i=1}^{M} \sum_{t=1}^{T}\left|\left(P_{i, g I}^{k}(t)-P_{i, g O}^{k}(t)\right)-\left(P_{i, g I}^{k-1}(t)-P_{i, g O}^{k-1}(t)\right)\right|
$$

When the changes of the user's payoff and power routine within the preset threshold in two successive iterations, the distributed optimization will be stopped and the equilibrium is achieved.

According to the distributed algorithm in Table 1, the user only needs to send its total power schedule to the utility control center and receive the electricity price over the control horizon. No communication is required among different users. Hence, the privacy of each user is protected effectively.

Table 1. Algorithm for distributed optimization algorithm for the energy scheduling of users.

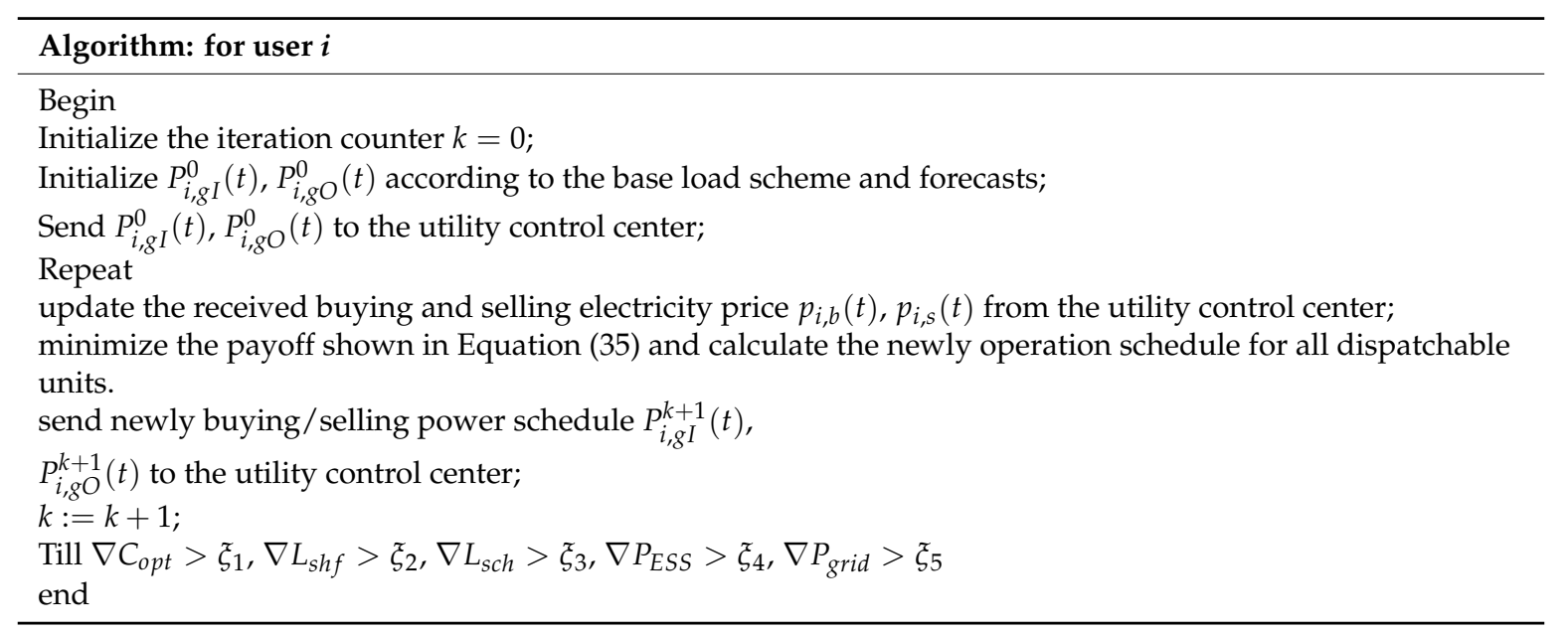

where $\nabla C_{o p t}, \nabla L_{s h f}, \nabla L_{s c h}, \nabla P_{E S S}, \nabla P_{\text {grid }}$ are the total cost difference, shift-able load power schedule difference, schedulable load power schedule difference, ESS power schedule difference and the buying/selling power schedule difference in two consecutive iterations, respectively.

In the distributed optimization algorithm, $\lambda_{i}^{k}$ is a user-defined parameter which affects the algorithm convergence. In this paper, $\lambda_{i}^{k}$ is defined based on the following considerations. First, the change of operation routines becomes small as the algorithm progresses. Second, the greater the change, the higher the penalty cost. Third, a small penalty coefficient is used for large power consuming users while a large penalty coefficient is used for small power consuming users. This is because high consuming users tend to care more about the electricity price. Correspondingly, the change of their operations impacts more on the electricity price. Specifically, $\lambda_{i}^{k}$ is computed as follows.

$$
\lambda_{i}^{k}=\pi \cdot\left(\left|\frac{\sum_{t=1}^{T} P_{u}^{k}(t)}{\sum_{t=1}^{T}\left(P_{i, g I}^{k}(t)+P_{i, g O}^{k}(t)\right)}\right|\right)^{1 / 2}
$$

The parameter $\pi$ is an auxiliary coefficient to adjust the convergence speed of the distributed optimization algorithm, and is set to 0.5 .

Nash equilibrium refers to the best solution to a non-cooperative game, that is, no player can gain more credits by changing his/her own strategy only [34]. In this paper, NE consists of a set of strategies $\left(y_{1}^{*}, y_{2}^{*}, \cdots, y_{i}^{*}, \cdots, y_{n}^{*}\right)$, when $i \in M, P\left(y_{i}^{*}, y_{-i}^{*}\right) \geq P\left(y_{i}, y_{-i}^{*}\right)$.

The game theory-based distributed user energy management strategy proposed in this paper satisfies the second fundamental welfare theorem [35] of the Walrasian equilibrium theory [36], this is because the base electricity price mechanism and game theory framework $\left(p_{u},\left\{P_{i}\right\}_{i=1}^{M}\right)$ meet the form of the Walrasian equilibrium theory. $p_{u}, P_{i}$ are the base electricity price and power schedule vector, respectively, and are defined as follows.

$$
P_{u}=\left\{P_{u}(1), P_{u}(2), \ldots, P_{u}(t), \ldots, P_{u}(T),\right\}
$$




$$
\begin{gathered}
P_{i}=\left\{P_{i}(1), P_{i}(2), \ldots, P_{i}(t), \ldots, P_{i}(T),\right\} \\
P_{i}(t)=\left\{P_{i, g I}(t), P_{i, g O}(t), P_{i, E S S d}(t), P_{i, E S S c}(t), l_{i, a} \delta_{i, a}^{k}(t), l_{i, b}^{k}(t)\right\}
\end{gathered}
$$

where $P_{i}$ is a vector with $\left(4+A_{i}+B_{i}\right) \times T$.

\subsection{MPC-Based Control Framework}

In recent years, the MPC framework has attracted many attention in power system energy management [26,31] due to its feedback and rolling horizon mechanism being suitable to handle varying information and stochastic parameters.

In this section, we formulate an MPC-based user energy management problem, whose solution yields a trajectory of control actions and states into the future that satisfies the dynamics and constraints of the user and smart grid operation while optimizing some given criteria. Only the first sample of the control actions is implemented, and then the horizon is shifted.

The detailed coordination procedure for MPC-based distributed optimization is as follow:

(i) At the end of period $\tau$, the EMS of user $i$ obtains the updated state of its related dispatchable units, including the energy level of ESS, $E_{i, E S S}(\tau)$, the operation status of shift-able loads, $\delta_{i, a}(\tau)$, the operation status, $\delta_{i, b}(\tau)$ and power demand, $l_{i, b}(\tau)$ of the schedulable loads. Then the EMS calculates the forecasted data of load demand, PV generation and wind production from period $\tau+1$ to $\tau+T$.

(ii) The distributed optimization model illustrated in Table 1 is solved individually for each user, reaching a Nash Equilibrium (NS), i.e., $\left(p_{u}^{*},\left\{P_{i}^{*}\right\}_{i=1}^{M}\right)$. The first sample of the control sequence is then sent to local controllers.

(iii) At the beginning of $\tau+1$, only the first sample of the control sequence is implemented. The insufficient power caused by forecast errors is compensated by the utility. On the contrary, the excess power will be sold back to the utility with a lower price. Finally, the EMS updates the parameters and forecast model with new data.

(iv) Go to step (i) until the end of the simulation.

The designed control variables for the MPC controller is $P_{i}$. The forecast error is $F E_{i}$ which contains $\left(2+F_{i}\right) \times T$ variables.

$$
F E_{i}=\left\{F E_{i, \text { wind }}, F E_{i, P V}, F E_{i, l B}, F E_{i, l f}\right\}
$$

It is expected that, by the MPC-based distributed optimization, an optimal plan can be obtained to potentially compensate the forecast errors.

\section{Simulation and Results}

In this section, we first compare our proposed MPC-based distributed DSM (DMPC) strategy with the traditional day-ahead programming-based distributed DSM (DDA) strategy [32]. Then, the impact of the penalty cost term of the shift-able and schedulable loads of the system operation routine is discussed. Finally, we compare our parallel distributed optimization framework with the sequential distributed optimization framework [16].

\subsection{Experiment Setup}

We consider a smart grid with four users. Each user has wind and PV generators, ESS unit and different kinds of smart loads. Related parameters like the capacity of PV and wind generators, the maximum base load demand and the maximum power interaction for each user is listed in Table 2 . The control and prediction horizon is $T=24 \mathrm{~h}$, the duration of each time interval is $1 \mathrm{~h}$.

The historical data of base load, wind and PV power generation for each user is collected and modified from Belgium's transmission system [37] as shown in Figure 2. The basic operation schedule 
is as follows. The shift-able loads and schedulable loads work as they are planed, no power is curtailed and no ESS is integrated. Since no specialized data set for power flexible loads, we only consider a total load demand for power flexible appliances, that is, $F=1$.

Also, we assume that the demand of flexible load is $30 \%$ of the base load in each period. The maximum curtailment ratios for the four users are preset as $[0.5,0.4,0.35,0.3]$, meanwhile, the discomfort penalty coefficients for curtailing the flexible loads are set as $[2.5,1.8,2.2,2]$.

There are 8 shift-able appliances for each user, the power demand, available operation time window, time needed to complete the task and the discomfort penalty coefficient are all listed in Table 3. Different discomfort penalty coefficients are set for different users since the user preferences are usually different. Similarly, the parameters for schedulable loads are shown in Table 4. Since the schedulable loads can adjust both the start time and operation power, two penalty cost coefficients are adopted.

The parameters of the ESS are listed in Table 5. The operation and maintenance cost for each unit $\mathrm{kWh}$ is $0.1 \$$. The charging/discharging efficiency for each ESS is 0.95 . The initial energy level for each ESS is half of its maximum energy level.

According to the rate-of-return regulations, we set the retail purchasing electricity price for each user at 1.2 times of the base price, and the retail selling price for each user is 0.8 times the base price. Furthermore, we set the extra power purchasing price in the real-time power compensation stage at 3 times the base price and 0.5 times of the base price to sell the extra power generation, due to the fact that the real-time adjustment of the utility needs more cost than a prescheduled plan. The cost parameters for the utility is $c_{1}=0.000066\left(\$ / k W h^{2}\right), c_{2}=0.18 \$ / k W h$. The capacity of the utility is 5 $M W$, and the minimum power output is $50 \mathrm{~kW}$. If the total power demand of all users is less than 50 $\mathrm{kW}$, the utility will only act as a servicer. In other words, the energy coordination should be performed among users themselves.

The parameters for the stopping criteria of the distributed optimization algorithm in Table 1 is set as follows.

$$
\xi_{1}=1, \xi_{2}=0.6, \xi_{3}=0.2, \xi_{4}=0.1, \xi_{5}=0.1
$$

Table 2. Power parameters for each user.

\begin{tabular}{ccccc}
\hline User & PV Plant & Wind Farm & PCC Node & Base Load \\
\hline 1 & 400 & 200 & 1200 & 800 \\
2 & 450 & 200 & 1000 & 600 \\
3 & 350 & 240 & 1200 & 700 \\
4 & 360 & 250 & 1500 & 900 \\
\hline
\end{tabular}

Table 3. Parameter of shift-able loads.

\begin{tabular}{ccccc}
\hline Shift-able Load & $\begin{array}{c}\text { Power Demand } \\
\mathbf{( k W )}\end{array}$ & $\begin{array}{c}\text { Operation Interval } \\
\mathbf{( h )}\end{array}$ & $\begin{array}{c}\text { Duration (h) } \\
\text { Coefficient (\$) }\end{array}$ \\
\hline Task 1 & 22 & $15-21$ & 2 & $0.1,0.32,0.42,0.32$ \\
Task 2 & 28 & $14-23$ & 4 & $0.4,0.34,0.34,0.24$ \\
Task 3 & 45 & $8-18$ & 6 & $0.35,0.25,0.29,0.15$ \\
Task 4 & 37.5 & $6-24$ & 8 & $0.28,0.18,0.15,0.25$ \\
Task 5 & 12 & $2-22$ & 12 & $0.35,0.24,0.26,0.26$ \\
Task 6 & 60 & $8-22$ & 7 & $0.34,0.35,0.35,0.37$ \\
Task 7 & 75 & $6-24$ & 9 & $0.36,0.17,0.36,0.19$ \\
Task 8 & 24 & $4-20$ & 4 & $0.27,0.35,0.35,0.34$ \\
\hline
\end{tabular}


Table 4. Parameters of schedulable loads.

\begin{tabular}{ccccccc}
\hline Load & $\begin{array}{c}\text { Base } \\
\text { Power }\end{array}$ & $\begin{array}{c}\text { Max, Min } \\
\text { Power }\end{array}$ & $\begin{array}{c}\text { Time } \\
\text { Window }\end{array}$ & Duration & Start Delay Penalty & $\begin{array}{c}\text { Power Change } \\
\text { Penalty }\end{array}$ \\
\hline Task 1 & 25 & 15,35 & $6-24$ & 9 & $0.2,0.27,0.34,0.26$ & $0.11,0.08,0.05,0.11$ \\
Task 2 & 56 & 15,105 & $6-16$ & 6 & $0.4,0.1,0.33,0.27$ & $0.05,0.1,0.1,0.07$ \\
Task 3 & 20 & 5,45 & $2-24$ & 12 & $0.25,0.36,0.37,0.25$ & $0.05,0.06,0.11,0.04$ \\
Task 4 & 45 & 15,105 & $4-24$ & 15 & $0.28,0.26,0.26,0.35$ & $0.1,0.06,0.056,0.05$ \\
Task 5 & 30 & 12,60 & $5-24$ & 13 & $0.36,0.15,0.28,0.37$ & $0.07,0.09,0.07,0.1$ \\
\hline
\end{tabular}

Table 5. Parameters of ESS.

\begin{tabular}{ccccc}
\hline & Max Charge/Discharge Power & Min Charge/Discharge Power & Max Energy Level & Min Energy Level \\
\hline user 1 & 160 & 5 & 320 & 64 \\
user 2 & 140 & 8 & 300 & 60 \\
user 3 & 120 & 6 & 260 & 50 \\
user 4 & 100 & 4 & 220 & 40 \\
\hline
\end{tabular}
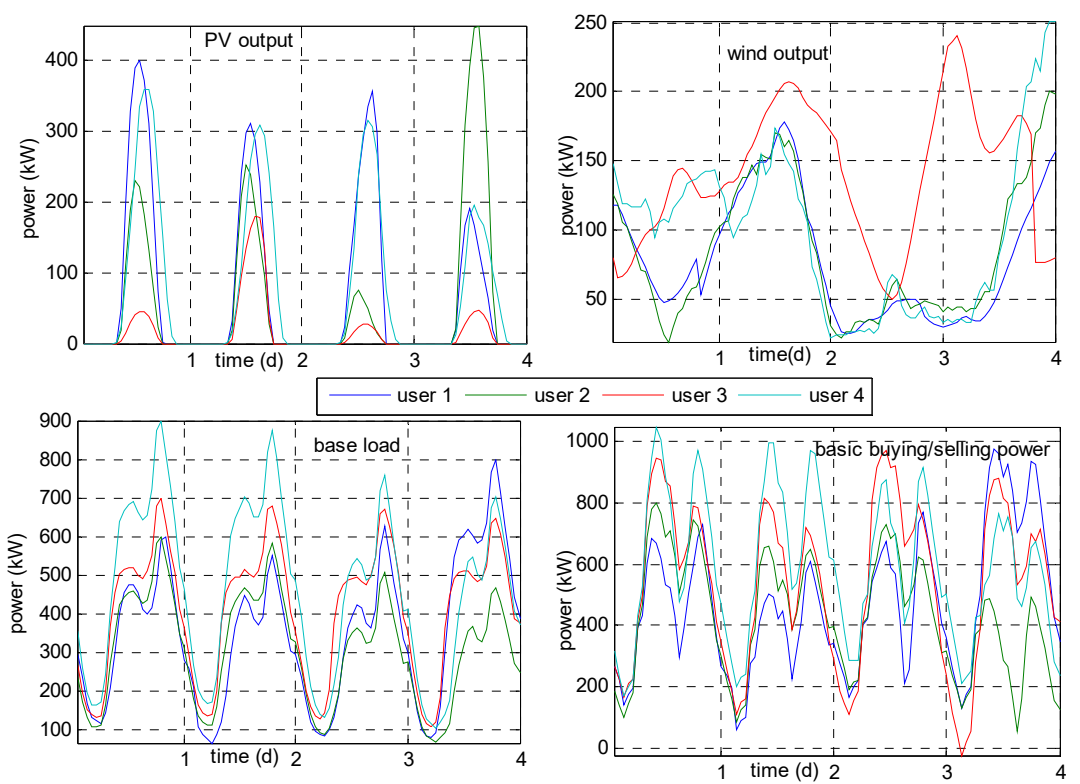

Figure 2. PV and wind generation, base load demand and the basic buy/selling power for each user.

\subsection{Simulation Results}

All simulations are run on a laptop with Intel(R) Core (TM) i5-3210M CPU @2.5 GHz and 8.00 GB memory. The ILOG's CPLEX v.12 optimization solver is utilized for solving the MIP models, MATLAB 2013a and YALMIP toolbox [38] are used for linking the CPLEX solver and computing the optimization model.

\subsubsection{Results of the DMPC Strategy and DDA Strategy}

First, the DDA strategy is introduced briefly. It is a traditional two-stage-based open-loop distributed energy management algorithm [15,32], including the scheduling stage and the real-time power compensation stage. The detailed process is shown as follow.

(i) At the scheduling stage, the EMS determines the operation schedule of the smart load appliances and the ESS over the control horizon by implementing the distributed optimization algorithm of Table 1 at the beginning of the day with the forecasts of RESs generation and load demand. The control sequence sent to the controllers of all dispatchable units should be implemented strictly. 
(ii) At the real-time power compensation stage, for each user the insufficient power will be provided by the utility company at a higher price and the extra power generation will be sold at a lower price back to the utility.

The operations for the DMPC and DDA at the real-time power compensation stage are the same, aiming to reduce the negative impacts introduced by forecast errors and randomness of the RESs output.

The routines of power generation for the utility under the DMPC and DDA are shown in Figure 3. Please note that the basic power refers to the utility generation where no optimization is implemented and no ESS unit is used. The scheduling power refers to the utility generation based on the forecasts at the scheduling stage. The real-time power refers to the utility generation adjusted with the real-time data at the real-time power compensation stage.

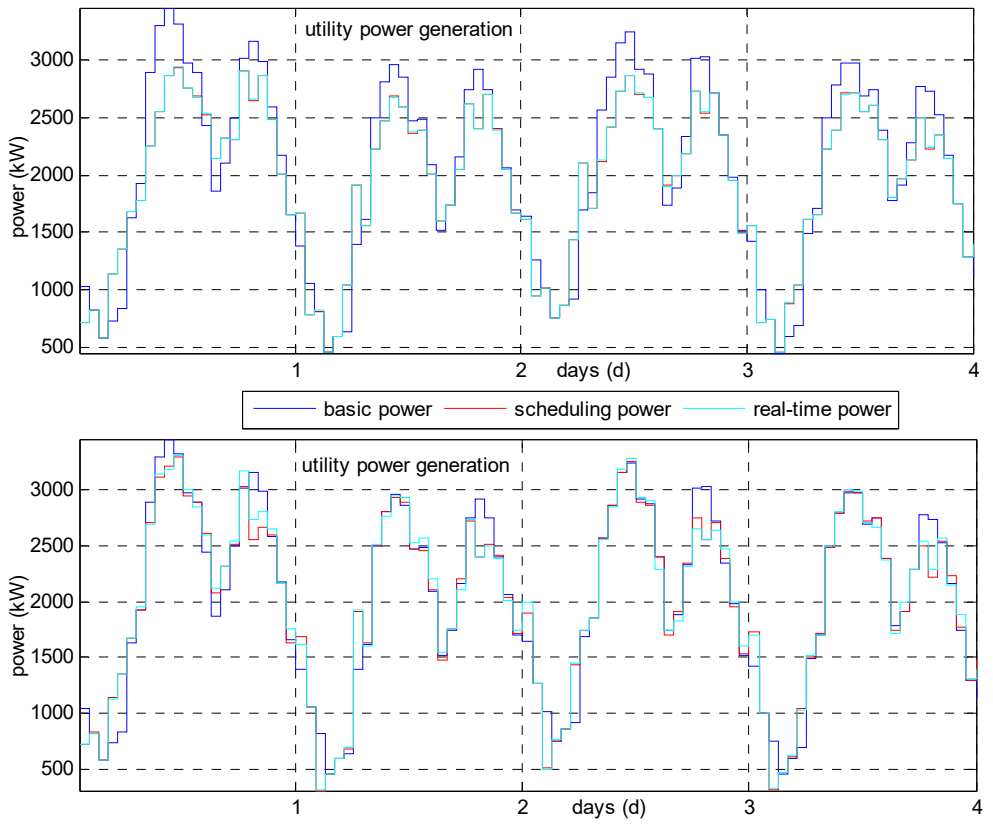

Figure 3. Utility generation for DMPC and DDA strategies in different cases.

The peak of the basic power is $3.459 \times 10^{3} \mathrm{~kW}$ in the 10 th hour of the first day, and its average value is $2.032 \times 10^{3} \mathrm{~kW}$. Thus, the peak-to-average ratio is 1.702 .

The power peak of the DMPC strategy at the scheduling stage is $2.934 \times 10^{3} \mathrm{~kW}$ at the 11 th hour of the first day, and its average power is $1.945 \times 10^{3} \mathrm{~kW}$. Thus, the peak-to-average ratio is 1.510 . Meanwhile, considering the forecast uncertainties, the real-time power generation of the utility at the real-time stage is different from that at the scheduling stage. The power peak of the DMPC strategy at real-time power compensation stage is $2.945 \times 10^{3} \mathrm{~kW}$ at the 11th hour of the first day, and its average power is $1.9455 \times 10^{3} \mathrm{~kW}$, and hence its peak-to-average ratio is 1.514 . Such results indicate that by the DMPC strategy, the impact caused by forecast uncertainties can be well handled. The peak-to-average ratio is reduced about $11.3 \%$ compared to the basic situation, and the peak power is reduced about $15 \%$ (more than $500 \mathrm{~kW}$ ). This demonstrates that the DMPC strategy can effectively save social welfare for the society.

The power peak of the DDA strategy at the scheduling stage is $3.292 \times 10^{3} \mathrm{~kW}$ at the 11th hour of the first day, and its average power is $2.018 \times 10^{3} \mathrm{~kW}$. Thus, the peak-to-average ratio is 1.63. This shows that the performance of the DDA strategy at the scheduling stage is inferior to that of the DMPC strategy. Moreover, the power peak of the DDA strategy at the real-time power compensation stage is $3.307 \times 10^{3} \mathrm{~kW}$ at the 11th hour of the first day, and its average power is $2.029 \times 10^{3} \mathrm{~kW}$. The peak-to-average ratio is thus 1.63. Compared with the DMPC strategy, the DDA strategy is inferior, 
reducing only $4.23 \%$ of the peak-to-average ratio, and $4.4 \%$ of the peak power (about $150 \mathrm{~kW}$ ) for the utility company

The reason for the inferior performance of DDA compared to the DMPC is that in DDA both the forecast and the operation schedule are made at the beginning of the day; however, the forecast and the operation schedule of the DMPC are adaptively adjusted every hour according to the newly updated information.

Figure 4 shows the power adjustment at the real-time stage under the DMPC and DDA strategy. From the figure, we can see that the amount of power adjustment with the DMPC strategy is much smaller than that with the DDA strategy. That is, the DDA is inferior to the DMPC strategy.
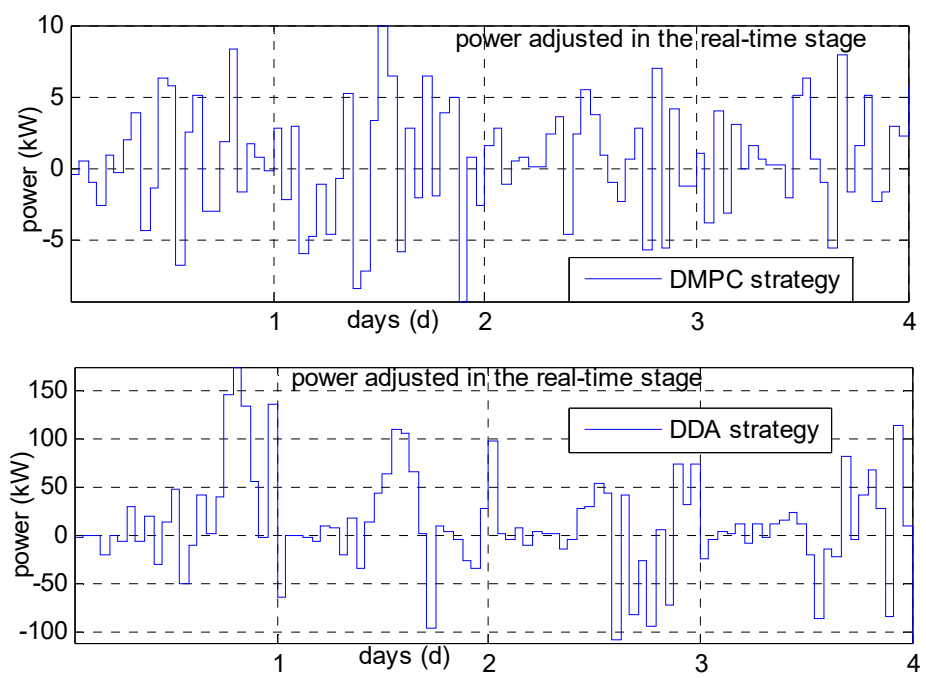

Figure 4. Power adjusted for DMPC and DDA strategies at real-time stage.

In addition to the comparison from the view of the utility, we next discuss the performance of the DMPC strategy from the view of users. The operation routines for each user and their dispatchable units with the DMPC strategy at the scheduling stage is illustrated in Figure 5.

User 1 buys $4.27 \times 10^{4} \mathrm{kWh}$ power energy from the utility while sells no power back to the utility company as power generated by RESs is always less than the load demand. User 1 spends $4.314 \times$ $10^{4} \$$ purchasing energy from the utility. The ESS charges $1.248 \times 10^{3} \mathrm{kWh}$ and discharges $1.127 \times$ $10^{3} \mathrm{kWh}$ power over the whole time horizon. The overall charged energy is a little larger than the discharged energy. This is because the power efficiency of the ESS is not $100 \%$. The operation and maintenance cost of the ESS cost is $237.5 \$$. Meanwhile, user 1 curtails $237 \mathrm{kWh}$ power flexible load demand, which results in a penalty cost of 533.8 \$. The starting time of shift-able appliances delayed about $10 \mathrm{~h}$, resulting in $0.39 \$$ penalty cost. The amount of energy adjustment for schedulable loads is $2.63 \times 10^{3} \mathrm{kWh}$, resulting in $54.124 \$$ penalty cost. Also, no schedulable appliance chooses to shift its operation time window.

User 2 buys $3.88 \times 10^{4} \mathrm{kWh}$ power energy from the utility while sells no power back to the utility company. $3.932 \times 10^{4} \$$ is spent to purchase energy from the utility. The ESS charges $1.239 \times 10^{3} \mathrm{kWh}$ and discharges $1.117 \times 10^{3} \mathrm{kWh}$ power over the horizon. The operation and maintenance cost of the ESS cost is $235.61 \$$. Meanwhile, user 2 curtails $88.01 \mathrm{kWh}$ power flexible load demand, resulting in $121.26 \$$ penalty cost. Since the discomfort penalty cost of user 2 is lower than that of user 1 , its penalty cost for curtailing loads is more effective than user 1 . The shift-able appliances delay about $7 \mathrm{~h}$, and the penalty cost for the delay is $0.335 \$$. Like user 1 , no schedulable appliance in user 2 chooses to shift its operation time window. The amount of energy adjustment for schedulable loads is $2.15 \times 10^{3} \mathrm{kWh}$, resulting in $47.76 \$$ penalty cost.

User 3 buys $5.0 \times 10^{4} \mathrm{kWh}$ power energy from the utility, and sells $15 \mathrm{kWh}$ power back to the utility company. The money spent to purchase energy from the utility is $5.191 \times 10^{4} \$$ while the 
revenue made by selling energy back to the utility is $4.2 \$$. The ESS charges $1.00 \times 10^{3} \mathrm{kWh}$ and discharges $0.916 \times 10^{3} \mathrm{kWh}$ power over the horizon. The operation and maintenance cost of the ESS is 191.89 \$. Meanwhile, user 3 curtails $92.182 \mathrm{kWh}$ power flexible load demand, resulting in 117.85\$ penalty cost. Compared with user 2, the power curtailment action for user 3 is more effective. This is because that both the penalty cost coefficient and the curtailed power of user 2 are lower than those of user 3 but the penalty cost of user 2 is higher than user 3 . The shift-able appliances delay about $8 \mathrm{~h}$, and the penalty cost is $0.265 \$$. Again, no schedulable appliance in user 3 chooses to shift the operation time window. The amount of energy adjustment for schedulable loads is $1.985 \times 10^{3} \mathrm{kWh}$, resulting in $45.125 \$$ penalty cost.

User 4 buys $5.526 \times 10^{4} \mathrm{kWh}$ power energy from the utility while sells no power back to the utility company. $5.594 \times 10^{4} \$$ is spent to purchase energy from the utility. The ESS charges $0.887 \times 10^{3} \mathrm{kWh}$ and discharges $0.7935 \times 10^{3} \mathrm{kWh}$ power over the horizon. The operation and maintenance cost of the ESS is 191.89 \$. User 4 curtails $90.51 \mathrm{kWh}$ power flexible load demand, resulting in 135.578 \$ penalty cost. The shift-able appliances delay about $14 \mathrm{~h}$, resulting in $0.46 \$$ penalty cost. No schedulable appliance in user 4 chooses to shift its operation time window. The amount of energy adjustment for schedulable loads is $1.508 \times 10^{3} \mathrm{kWh}$, resulting in $31.98 \$$ penalty cost.

Though the rolling horizon and feedback mechanism can reduce the negative impact introduced by the random and intermittent outputs of RESs to some extent, the forecast uncertainties cannot be completely due to the forecast model is imperfect. Therefore, user 1 must purchase $67.19 \mathrm{kWh}$ energy from the utility at a high electricity price with a total cost $169.97 \$$. Correspondingly, user 1 sells $55.48 \mathrm{kWh}$ energy back to the utility at a low electricity price, making revenue of $22.67 \$$. User 2 must purchase $72.03 \mathrm{kWh}$ energy from the utility at a total cost $182.14 \$$. Correspondingly, user 2 sells $45.41 \mathrm{kWh}$ energy back to the utility, making revenue of $18.3 \$$. Due to the penetration level of RESs output in user 3 is the highest, the influence of the forecast uncertainty is the highest. User 3 has to purchase more energy, i.e., $93.72 \mathrm{kWh}$ from the utility with a total cost of $231.62 \$$. Correspondingly, user 3 sells $72.5 \mathrm{kWh}$ energy back to the utility, making revenue of $27.83 \$$. User 4 purchases $71.53 \mathrm{kWh}$ energy from the utility with a total cost of $175.8 \$$. Correspondingly, user 4 sells $78.66 \mathrm{kWh}$ energy back to the utility, making revenue of $31.85 \$$.
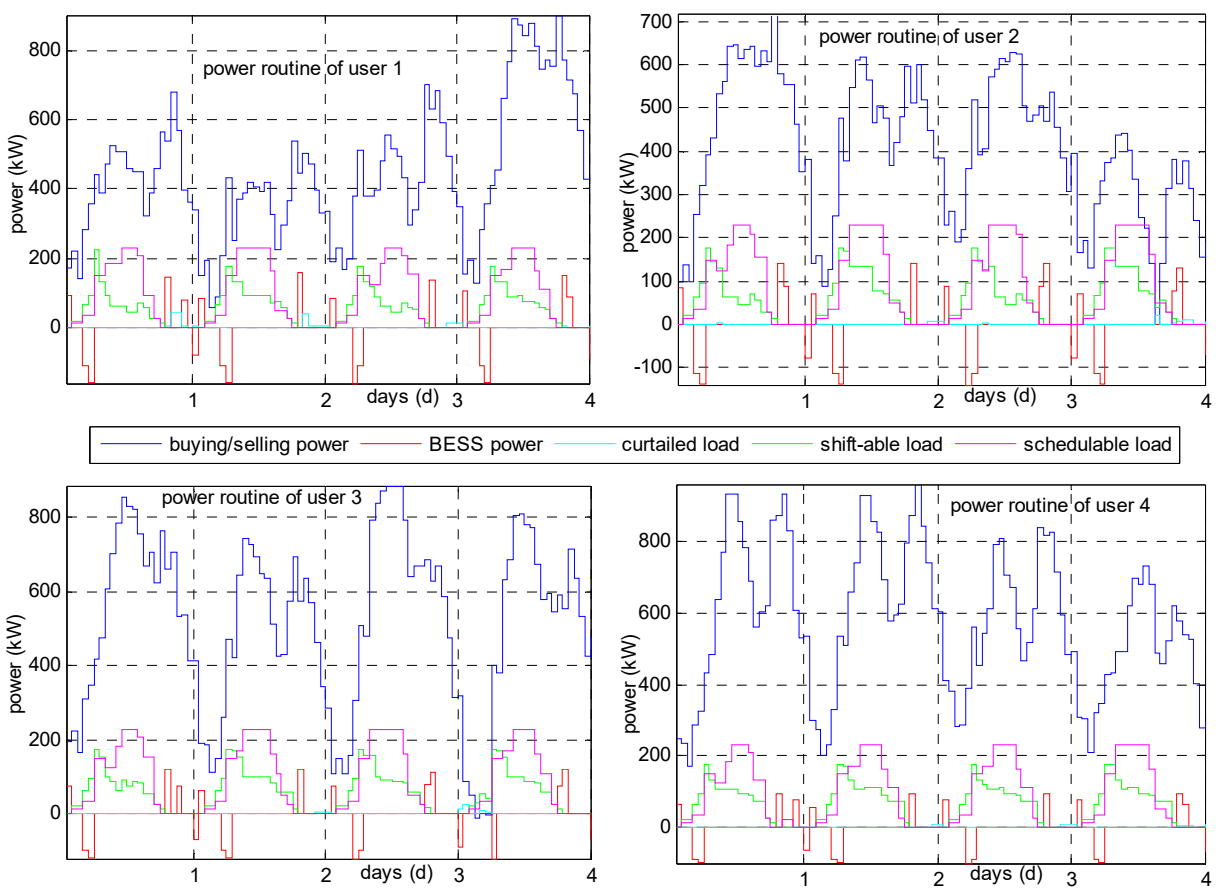

Figure 5. Power routine for each user and each dispatchable units with DMPC strategy. 
The operation costs for the DPMC and DDA strategies at the scheduling and real-time power compensation stages are shown in Table 6. From the results, we can find that the operation cost of the DMPC and DDA is nearly the same at the scheduling stage while different at the real-time stage (the cost by the DDA is higher than that by the DMPC). This is because the forecasted electricity prices for the two strategies at the scheduling stage are similar. However, at the real-time stage, the closed-loop-based DMPC can adjust control actions with newly updated forecasts while the open-loop-based DDA cannot. This leads DDA to cost more.

Table 6. Operation costs for each user with different strategies.

\begin{tabular}{ccccc}
\hline User & $\begin{array}{c}\text { Scheduling Cost for } \\
\text { MPC Strategy (\$) }\end{array}$ & $\begin{array}{c}\text { Adjustment Cost for } \\
\text { MPC Strategy (\$) }\end{array}$ & $\begin{array}{c}\text { Scheduling Cost for } \\
\text { DDA Strategy (\$) }\end{array}$ & $\begin{array}{c}\text { Adjustment Cost for } \\
\text { DDA Strategy (\$) }\end{array}$ \\
\hline User 1 & $4.876 \times 10^{4}$ & 147.3 & $4.876 \times 10^{4}$ & $2.158 \times 10^{3}$ \\
User 2 & $4.390 \times 10^{4}$ & 163.847 & $4.400 \times 10^{4}$ & $1.415 \times 10^{3}$ \\
User 3 & $5.690 \times 10^{4}$ & 203.79 & $5.692 \times 10^{4}$ & $1.74 \times 10^{3}$ \\
User 4 & $6.023 \times 10^{4}$ & 143.954 & $6.023 \times 10^{4}$ & $2.727 \times 10^{3}$ \\
\hline
\end{tabular}

\subsubsection{Analysis of the Penalty Cost Term for the Utility Power Generation}

Figure 6 presents the utility power generation routine with and without considering the penalty cost term. We use the term no penalty to refer to the case that no penalty cost is considered. Under the no penalty case, the peak power is $2.898 \times 10^{3} \mathrm{~kW}$, and the average power is $1.872 \times 10^{3} \mathrm{~kW}$. The peak-to-average ratio is thus 1.548. Though the peak power and the average power are both lower than the case considering penalty cost, the peak-to-average for no penalty case is higher than penalty case. This clearly shows that penalty cost term has important impact on the utility.

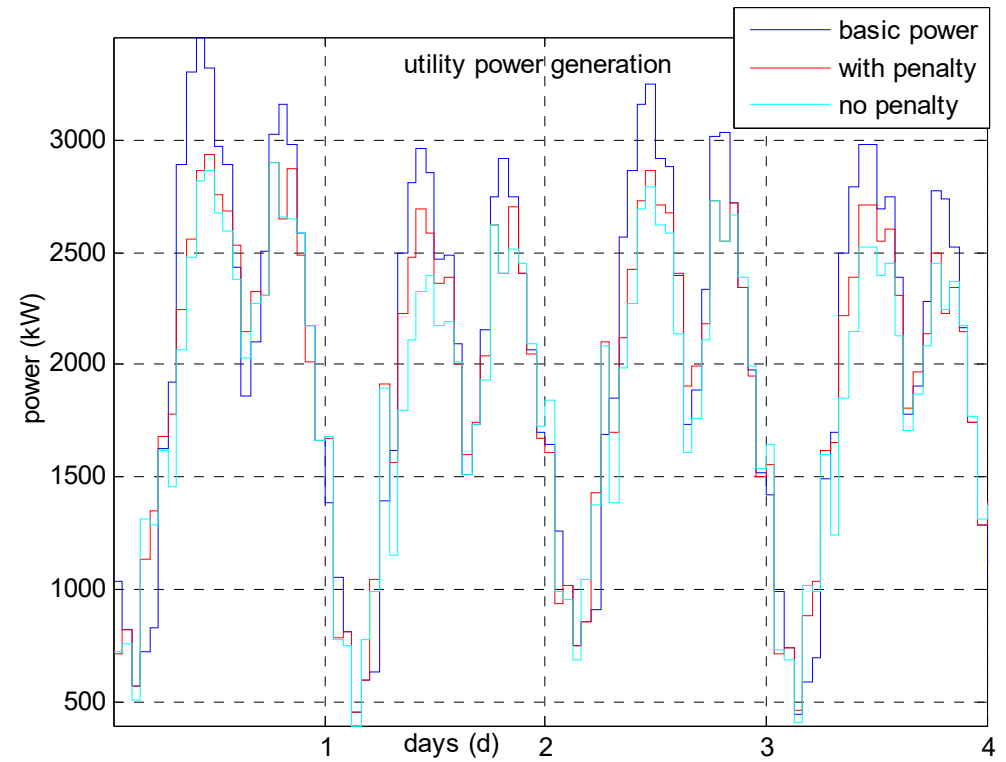

Figure 6. Power generation of the utility with or without considering penalty cost terms.

\subsubsection{Comparison of the Parallel and Sequential Optimization Algorithm}

This section compares the performance of the sequential distributed optimization (SDO) algorithm [16] with the parallel distributed optimization (PDO) algorithm described in Table 1. The users apply the SDO algorithm to update their operation schedule sequentially, namely, the electricity price has to change after the optimization scheduling. The first day's data is used to present the comparison results as shown in Figures 7 and 8. 

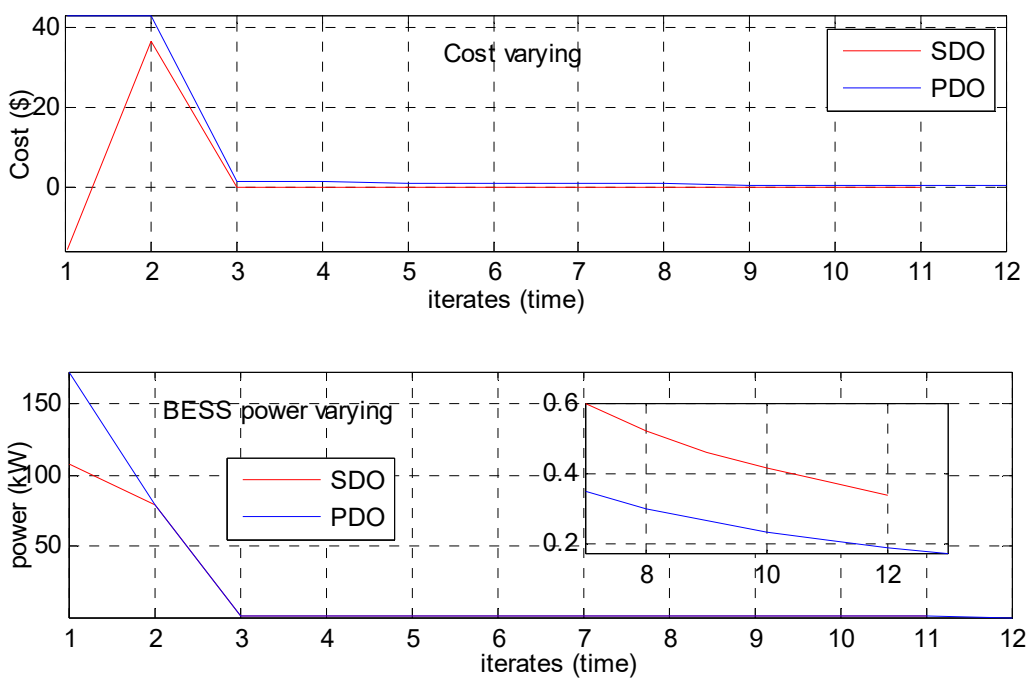

Figure 7. Utility fuel cost and BESS power change for the PDO and SDO algorithms.

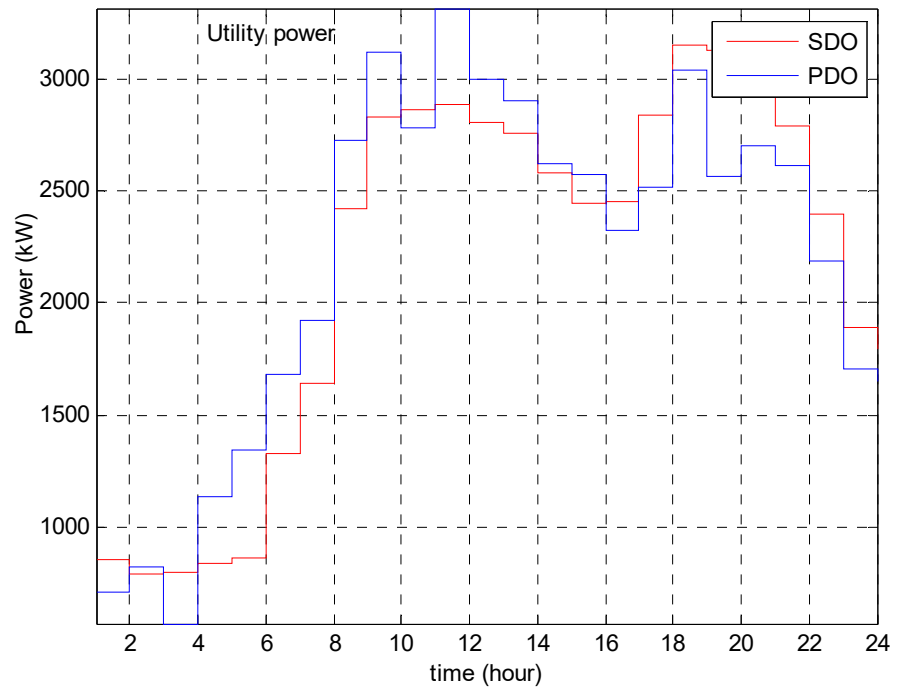

Figure 8. Utility power of the first day for PDO and SDO algorithms.

Figure 7 shows that the SDO algorithm needs to run 11 iterations to achieve an equilibrium for a user. Since there are four users, so the total required iterations are 44 . However, the PDO algorithm reaches an equilibrium after 12 iterations. This indicates that the PDO algorithm converges much faster than the SDO algorithm. Moreover, it can be observed from Figure 8 that the peak power of the PDO algorithm is only a bit higher than the SDO algorithm. This further demonstrates the advantage of the PDO over the SDO algorithm.

\section{Conclusions}

In this study, an MPC-based distributed demand side management strategy is proposed to provide optimal control actions for energy users in a smart grid. The users are equipped with renewable energy source (RES) generators, energy storage system (ESS) units and different kinds of smart loads. For each user, an energy management system (EMS) is used to determine the operation scheme of dispatchable units and the interaction with the utility (i.e., purchasing power from or selling power back to the utility). Experimental results show that the proposed MPC-based distributed DSM (DMPC) strategy enables users to optimally control their own subsystems individually, and coordinate them properly when necessary. Moreover, its performance is demonstrated to be better than the traditional day-ahead programming-based distributed DSM (DDA) strategy [32]. In addition, 
the proposed parallel distributed optimization method is also demonstrated to be superior to the sequential distributed optimization algorithm.

With respect to the future work, we would like to analyze the games among the users and the utility. Also, the convergence property of the parallel distributed optimization method needs to be theatrically analyzed.

Author Contributions: Conceptualization, R.W.; Methodology, M.H.; Software, J.C.; Validation, Y.H., and F.Z.; Formal Analysis, M.H.; Writing-Original Draft Preparation, M.H.; Writing-Review \& Editing, R.W., J.W. and F.Z.

Funding: This research was funded by by the National Natural Science Foundation of China (No.61773390), the Outstanding Natural Science Foundation of Hunan Province (2017JJ1001), the Hunan Youth elite program(2018RS3081), the key project of National University of Defense Technology (ZK18-02-09), the Natural Science Foundation of Guangdong Province (No. 2018A030313258) and the Project of Internation as well as Hongkong, Macao $\backslash \&$ Taiwan Science and Technology Cooperation Innovation Platform in Universities in Guangdong Province (2015KGJHZ023).

Conflicts of Interest: The authors declare no conflict of interest.

\section{References}

1. Strbac, G. Demand side management: Benefits and challenges. Energy Policy 2008, 36, 4419-4426. [CrossRef]

2. Zhao, N.; Yu, F.R.; Sun, H. Adaptive energy-efficient power allocation in green interference-alignment-based wireless networks. IEEE Trans. Veh. Technol. 2015, 64, 4268-4281. [CrossRef]

3. Palensky, P.; Dietrich, D. Demand side management: Demand response, intelligent energy systems, and smart loads. IEEE Trans. Ind. Inform. 2011, 7, 381-388. [CrossRef]

4. Meng, F.L.; Zeng, X.J. A Profit Maximization Approach to Demand Response Management with Customers Behavior Learning in Smart Grid. IEEE Trans. Smart Grid 2016, 7, 1516-1529. [CrossRef]

5. Ye, F.; Qian, Y.; Hu, R.Q. A real-time information based demand-side management system in smart grid. IEEE Trans. Parallel Distrib. Syst. 2016, 27, 329-339. [CrossRef]

6. Beaudin, M.; Zareipour, H. Home energy management systems: A review of modelling and complexity. Renew. Sustain. Energy Rev. 2015, 45, 318-335. [CrossRef]

7. Shariatzadeh, F.; Mandal, P.; Srivastava, A.K. Demand response for sustainable energy systems: A review, application and implementation strategy. Renew. Sustain. Energy Rev. 2015, 45, 343-350. [CrossRef]

8. Zhang, Y.; Zhang, T.; Wang, R.; Liu, Y.; Guo, B. Optimal operation of a smart residential microgrid based on model predictive control by considering uncertainties and storage impacts. Sol. Energy 2015, 122, 1052-1065. [CrossRef]

9. Prodan, I.; Zio, E. A model predictive control framework for reliable microgrid energy. Electr. Power Energy Syst. 2014, 61, 399-409. [CrossRef]

10. Soliman, H.M.; Leon-Garcia, A. Game-theoretic demand-side management with storage devices for the future smart grid. IEEE Trans. Smart Grid 2014, 5, 1475-1485. [CrossRef]

11. $\mathrm{Bu}, \mathrm{S}$; $\mathrm{Yu}, \mathrm{F} . \mathrm{R}$. A game-theoretical scheme in the smart grid with demand-side management: Towards a smart cyber-physical power infrastructure. IEEE Trans. Emerg. Top. Comput. 2013, 1, 22-32. [CrossRef]

12. Aalami, H.; Yousefi, G.; Moghadam, M. Demand response model considering EDRP and TOU. In Proceedings of the 2008 IEEE/PES Transmission and Distribution Conference and Exposition, Chicago, IL, USA, 21-24 April 2008.

13. Setlhaolo, D.; Xia, X.; Zhang, J. Optimal scheduling of household appliances for demand response. Electr. Power Syst. Res. 2014, 116, 24-28. [CrossRef]

14. Zhang, Y.; Wang, R.; Zhang, T.; Liu, Y.; Guo, B. Model predictive control-based operation management for a residential microgrid with considering forecast uncertainties and demand response strategies. IET Gener. Transm. Distrib. 2016, 10, 2367-2378. [CrossRef]

15. Erdinc, O. Economic impacts of small-scale own generating and storage units, and electric vehicles under different demand response strategies for smart households. Appl. Energy 2014, 126, 142-150. [CrossRef]

16. Mohsenian-Rad, A.H.; Wong, V.W.; Jatskevich, J.; Schober, R.; Leon-Garcia, A. Autonomous demand-side management based on game-theoretic energy consumption scheduling for the future smart grid. IEEE Trans. Smart Grid 2010, 1, 320-331. [CrossRef] 
17. Song, L.; Xiao, Y.; van der Schaar, M. Demand side management in smart grids using a repeated game framework. IEEE J. Sel. Areas Commun. 2014, 32, 1412-1424. [CrossRef]

18. Fathi, M.; Bevrani, H. Adaptive Energy Consumption Scheduling for Connected Microgrids under Demand Uncertainty. IEEE Trans. Power Deliv. 2013, 28, 1576-1583. [CrossRef]

19. Tan, Z.; Yang, P.; Nehorai, A. Distributed demand response for plug-in electrical vehicles in the smart grid. In Proceedings of the 2013 IEEE 5th International Workshop on Computational Advances in Multi-Sensor Adaptive Processing (CAMSAP), St. Martin, France, 15-18 December 2013; pp. 468-471.

20. Atzeni, I.; Ordóñez, L.G.; Scutari, G.; Palomar, D.P.; Fonollosa, J.R. Noncooperative and cooperative optimization of distributed energy generation and storage in the demand-side of the smart grid. IEEE Trans. Signal Process. 2013, 61, 2454-2472. [CrossRef]

21. Yang, P.; Chavali, P.; Gilboa, E.; Nehorai, A. Parallel Load Schedule Optimization with Renewable Distributed Generators in Smart Grids. IEEE Trans. Smart Grid 2013, 4, 1431-1441. [CrossRef]

22. Kamyab, F.; Amini, M.; Sheykhha, S.; Hasanpour, M.; Jalali, M.M. Demand response program in smart grid using supply function bidding mechanism. IEEE Trans. Smart Grid 2016, 7, 1277-1284. [CrossRef]

23. Wu, C.; Mohsenian-Rad, H.; Huang, J.; Wang, A.Y. Demand side management for wind power integration in microgrid using dynamic potential game theory. In Proceedings of the 2011 IEEE GLOBECOM Workshops (GC Wkshps), Houston, TX, USA, 5-9 December 2011; pp. 1199-1204.

24. Wang, Z.; Chen, B.; Wang, J.; Begovic, M.M.; Chen, C. Coordinated Energy Management of Networked Microgrids in distribution systems. IEEE Trans. Smart Grid 2015, 6, 45-53. [CrossRef]

25. Stephens, E.R.; Smith, D.B.; Mahanti, A. Game theoretic model predictive control for distributed energy demand-side management. IEEE Trans. Smart Grid 2015, 6, 1394-1402. [CrossRef]

26. Chen, C.; Wang, J.; Heo, Y.; Kishore, S. MPC-Based Appliance Scheduling for Residential Building Energy Management Controller. IEEE Trans. Smart Grid 2013, 4, 1401-1410. [CrossRef]

27. Kriett, P.O.; Salani, M. Optimal control of a residential microgrid. Energy 2012, 42, 321-330. [CrossRef]

28. Arriagada, E.; López, E.; Roa, C.; López, M.; Vannier, J.C. A Stochastic Economic Dispatch Model with Renewable Energies Considering Demand and Generation Uncertainties. In Proceedings of the 2013 IEEE Grenoble Conference, Grenoble, France, 16-20 June 2013; pp. 1-6.

29. Wang, M.Q.; Gooi, H.B. Spinning reserve estimation in micro grids. IEEE Trans. Power Syst. 2011, 26, 1164-1174. [CrossRef]

30. Li, F.; Zhang, L.Z.; Wang, Y.Y.; Feng, J.S. Research on wind power integration capacity of hydro-thermal-wind power system with energy storage system. Appl. Mech. Mater. 2014, 448, 2309-2315. [CrossRef]

31. Parisio, A.; Rikos, E.; Glielmo, L. A model predictive control approach to microgrid operation optimization. IEEE Trans. Control Syst. Technol. 2014, 22, 1813-1827. [CrossRef]

32. Chavali, P.; Yang, P.; Nehorai, A. A distributed algorithm of appliance scheduling for home energy management system. IEEE Trans. Smart Grid 2014, 5, 282-290. [CrossRef]

33. Netz, J.S. Price regulation: A (non-technical) overview. Encycl. Law Econ. 2000, 3, 1396-1465.

34. Osborne, M.J.; Rubinstein, A. A Course in Game Theory; MIT Press: Cambridge, MA, USA, 1994.

35. Li, N.; Chen, L.; Low, S.H. Optimal demand response based on utility maximization in power networks. In Proceedings of the 2011 IEEE Power and Energy Society General Meeting, Detroit, MI, USA, 24-29 July 2011; pp. 1-8.

36. Weber, T.A. Oxford Handbook on Pricing Management; Oxford University Press: New York, NY, USA, 2010.

37. Available online: http://www.elia.be/en/about-elia (accessed on 16 December 2018).

38. Löfberg, J. YALMIP: A toolbox for modeling and optimization in MATLAB. In Proceedings of the 2004 IEEE International Symposium, New Orleans, LA, USA, 2-4 September 2004; pp. 284-289.

(C) 2019 by the authors. Licensee MDPI, Basel, Switzerland. This article is an open access article distributed under the terms and conditions of the Creative Commons Attribution (CC BY) license (http:/ / creativecommons.org/licenses/by/4.0/). 